\title{
Unsteady Free Convective Heat and Mass Transfer Past a Vertical Cone in Non-Darcian Porous Media
}

\author{
S. Gouse Mohiddin \\ Department of Mathematics, \\ Madanapalle Institute of \\ Technology \& Science, \\ Madanapalle, AP, India
}

\author{
O. Anwar Bég \\ Mechanical Engineering \\ Subject Group, Sheffield \\ Hallam University, Sheffield, \\ S1 1WB, England, UK
}

\author{
S. Vijaya Kumar Varma \\ Department of Mathematics, \\ Sri Venkateswara University, \\ Tirupati - 517502,
}

AP, India

\begin{abstract}
A numerical solution of transient laminar free convective heat and mass transfer in a viscoelastic fluid past a vertical cone in non-Darcian porous media in the presence of thermal radiation is presented. The Walters-B liquid model is employed to simulate medical creams and other rheological liquids encountered in biotechnology and chemical engineering. This rheological model introduces supplementary terms into the momentum conservation equation. The dimensionless governing equations of the flow are solved by an implicit finite difference scheme of Crank-Nicolson type. The velocity, temperature and concentration fields have been studied for the effect of radiation parameter, viscoelasticity parameter, Prandtl number, Schmidt number, buoyancy ratio parameter, Darcy number, Grashof number, Forchheimer number and semi vertical angle. The local skin friction, Nusselt number and Sherwood number are also presented and analyzed graphically. The numerical results are validated by comparisons with previously published work and are found to be in excellent agreement.
\end{abstract}

\section{Keywords}

Vertical Cone; Free convection, porous media; CrankNicolson method; Forchheimer number; viscoelasticity

\section{NOMENCLATURE}

$\mathrm{x}, \mathrm{y} \quad$ coordinates along the cone generator and normal to the generator

$\begin{array}{ll}\mathrm{u}, \mathrm{v} & \text { velocity components along the } \mathrm{x} \text { - and } \mathrm{y} \text {-directions } \\ \mathrm{g} & \text { gravitational acceleration } \\ \mathrm{r} & \text { local radius of cone } \\ t^{\prime} & \text { time } \\ \mathrm{t} & \text { dimensionless time } \\ T^{\prime} & \text { temperature } \\ \mathrm{T} & \text { dimensionless temperature } \\ C^{\prime} & \text { concentration } \\ \mathrm{C} & \text { dimensionless concentration } \\ \mathrm{D} & \text { mass diffusion coefficient } \\ \mathrm{K} & \text { permeability of porous medium }\end{array}$

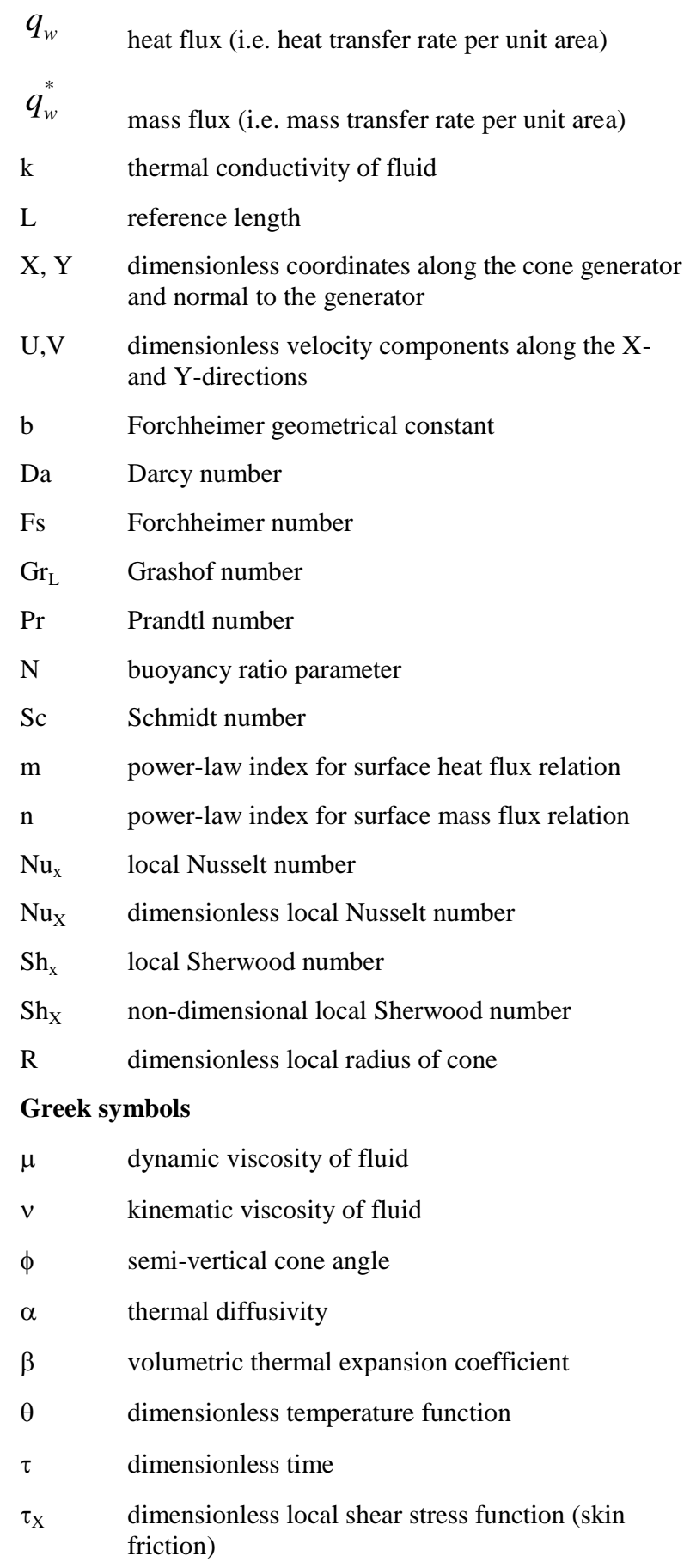




\section{Subscripts}

W

condition on the wall

$\infty \quad$ free stream condition

\section{INTRODUCTION}

Many transport processes can be found in various ways in both nature and technology, in which the combined heat and mass transfer occur due to buoyancy forces caused by thermal diffusion and mass diffusion. In recent years, the problem of radiation effect on convection boundary layer flow has caused much attention because of the important applications in space technology and processes involving high temperatures. Nuclear power plants, gas turbines and the various propulsion devices for air craft, missiles and space vehicles are examples of such engineering areas. Several studies have examined theoretically the effect of thermal radiation on viscoelastic convection flows. The Rosseland diffusion flux model and a short-memory (Walters-B) non-Newtonian model was used in [1] to study the effect of elastic parameter and thermal radiation on viscoelastic flow. The effect of radiation on natural convection about an isothermal truncated cone was investigated in [2]. Viscoelastic flows and transport phenomena arise in numerous areas of chemical, industrial process, biosystems, food processing and biomedical engineering. These include the rheology of liquid crystal precursors employed in the manufacture of carbon superfibers, crude oil emulsion processing, paper coating rheological processing, propulsive ciliary transport of respiratory airway mucus, thermocapillary bubble dynamics in weakly elastic fluids, rheo-reactor phosphatation flows, flour rheology, mayonnaise elastico-viscous flows, xanthan gum hydrogel flows, polygalacturonase-based food stuff.

Darcy's Law has been used exclusively in studies of porous-medium flow in reservoirs, however, there is considerable evidence that high-velocity non-Darcy flow occurs in many subsurface systems, such as in the flow near wells of oil or gas production, groundwater pumping, and liquid waste injection. Darcy's law, describing a linear relationship between volumetric flow rate (Darcy velocity) and pressure (or potential) gradient, has been the fundamental principle in analyzing flow processes in porous media. There are a few studies about the natural convection from cones in porous media saturated with Newtonian and non-Newtonian fluids. Reference [3] studied the problem of natural convection of a Darcian fluid about a cone. The coupled heat and mass transfer by free convection over truncated cone in porous media for variable wall temperature and concentration as well as for variable heat flux and variable mass flux was examined in [4]. Reference [5] used integral method to study heat and mass transfer by natural convection from truncated cones in porous media with variable wall temperature and concentration. The problem of natural convection of a nonNewtonian fluid from a vertical truncated cone was studied in [6] with variable wall temperature and concentration. In analyzing non-Darcy flow through porous media, the Forchheimer equation has been exclusively used to describe non-Darcy porous media flow, and has been extended to multiphase flow conditions. Recent laboratory studies and analyses have shown that the Barree and Conway model [7] is able to describe the entire range of relationships between flow rate and potential gradient from low to high flow rates through porous media, including those in transitional zones. Overall, this work provides an improved platform for modeling multiphase non-Darcy flow in oil and gas reservoirs, including complex fractured systems such as shale gas reservoirs.

The above studies did not consider combined viscoelastic momentum, heat and mass transfer from a vertical cone, either for the steady case or unsteady case in the presence of thermal radiation. Owing to the significance of this problem in chemical technological processing the transient case of such a flow is considered in this paper.

\section{CONSTITUTIVE EQUATIONS FOR THE WALTERS-B VISCOELASTIC MODEL}

$$
\begin{aligned}
& p_{i k}=-p g_{i k}+p_{i k}^{*} \\
& p_{i k}^{*}=2 \int_{-\infty}^{t} \Psi\left(t-t^{*}\right) e_{i k}^{(1)}\left(t^{*}\right) d t^{*} \\
& \Psi\left(t-t^{*}\right)=\int_{0}^{\infty} \frac{N(\tau)}{\tau} e^{\left(-\left(t-t^{*}\right) / \tau\right)} d \tau
\end{aligned}
$$

where $p_{i k}$ is the stress tensor, $\mathrm{p}$ is arbitrary isotropic pressure, $g_{i k}$ is the metric tensor of a fixed coordinate system $\mathrm{x}_{\mathrm{i},}, e_{i k}^{(1)}$ is the rate of strain tensor and $N(\tau)$ is the distribution function of relaxation times, $\tau$. The following generalized form of (2) has been shown in [8] to be valid for all classes of motion and stress.

$$
p^{* i k}(x, t)=2 \int_{-\infty}^{t} \Psi\left(t-t^{*}\right) \frac{\partial x^{i}}{\partial x^{*} m} \frac{\partial x^{k}}{\partial x^{* r}} e^{(1) m r}\left(x^{* *} t^{*}\right) d t^{*}
$$

in which $x_{i}^{*}=x_{i}^{*}\left(x, t, t^{*}\right)$ denotes the position at time $\mathrm{t}^{*}$ of the element which is instantaneously at the position, $x_{i}$, at time, $t$. Liquids obeying the relations (1) and (4) are of the Walters-B' type. For such fluids with short memory i.e. low relaxation times, equation (4) may be simplified to:

$$
p^{* i k}(x, t)=2 \eta_{0} e^{(1) i k}-2 k_{0} \frac{\partial e^{(1) i k}}{\partial t}
$$

in which $\quad \eta_{0}=\int_{0}^{\infty} N(\tau) d \tau$ defines the limiting Walters-B' viscosity at low shear rates, $k_{0}=\int_{0}^{\infty} \tau N(\tau) d \tau$ is the WaltersB' viscoelasticity parameter and $\frac{\partial}{\partial t}$ is the convected time derivative. This rheological model is very versatile and robust and provides a relatively simple mathematical formulation which is easily incorporated into boundary layer theory for engineering applications

\section{MATHEMATICAL MODEL}

An axi-symmetric unsteady laminar free convection heat and mass transfer in a viscoelastic, incompressible, absorbing-emitting, non-scattering, optically-thick fluid from an isothermal vertical cone with uniform surface temperature and concentration in a Darcy-Forchheimer fluid saturated isotropic porous medium in the presence of thermal radiation 
is considered. Unsteady incompressible flow and non-reactive mass diffusion in a free convective viscoelastic flow driven by species buoyancy force occurs upwards along the cone. In addition, the formulation of mathematical equations is based on the following assumptions:

- The physical properties of the fluid are constant except density in the buoyancy force term in the momentum equation.

- The density is a linear function of temperature and species concentration given by $\rho=\rho_{0}\left[1-\beta^{*}\left\{\left(T^{\prime}-T_{0}^{\prime}\right)+\beta\left(C^{\prime}-C_{0}^{\prime}\right)\right\}\right]$.

Hence, the Boussinesq's approximation is taken into account for the buoyancy effects induced by thermal and mass diffusion.

- Viscous dissipation effects and pressure gradient along the boundary layer are negligible.

- The magnetic Reynolds number is very small so that the induced magnetic field is negligible.

- The magnetic field is not strong enough to cause Joule heating so that the term due to electrical dissipation is neglected in energy equation.

- The concentration $C^{\prime}$ of the diffusing species in the binary mixture is assumed to be very less in comparison to the other chemical species, which are present. This leads to the assumption that the Soret and Dufour effects are negligible.

- The chemical reaction in the fluid is also negligible.

Also implicit in our analysis is the assumption that the cone surface and the surrounding fluid which are at rest possess the same temperature $T_{\infty}^{\prime}$ and concentration level $C_{\infty}^{\prime}$. At time $t^{\prime}>0$, the temperature of the cone surface and the concentration level near the cone surface are elevated and are sustained as constant thereafter. The co-ordinate system chosen (as shown in Fig.1) is such that the $x$-coordinate is directed along the surface of the cone from the apex $(x=0)$ and the $y$-coordinate is orientated perpendicular to this i.e. at right angles to the cone surface, outwards. Here, $\phi$ designates the semi-vertical angle of the cone and $r$ is the local radius of the cone. Under the above assumptions, implementing the shear-stress strain tensor for a Walters-B liquid, the appropriate unsteady incompressible conservation equations for the regime may be shown to take the form:

$$
\frac{\partial(u r)}{\partial x}+\frac{\partial(v r)}{\partial y}=0
$$

$\frac{\partial u}{\partial t^{\prime}}+u \frac{\partial u}{\partial x}+v \frac{\partial u}{\partial y}=v \frac{\partial^{2} u}{\partial y^{2}}-k_{0} \frac{\partial^{3} u}{\partial y^{2} \partial t^{\prime}}$

$+g \beta \cos \phi\left(T^{\prime}-T_{\infty}^{\prime}\right)+g \beta^{*} \cos \phi\left(C^{\prime}-C_{\infty}^{\prime}\right)-\frac{v}{K} u-\frac{b}{K} u^{2}$

$\frac{\partial T^{\prime}}{\partial t^{\prime}}+u \frac{\partial T^{\prime}}{\partial x}+v \frac{\partial T^{\prime}}{\partial y}=\alpha \frac{\partial^{2} T^{\prime}}{\partial y^{2}}-\frac{1}{\rho c_{p}} \frac{\partial q_{r}}{\partial y}$ $\frac{\partial C^{\prime}}{\partial t^{\prime}}+u \frac{\partial C^{\prime}}{\partial x}+v \frac{\partial C^{\prime}}{\partial y}=D \frac{\partial^{2} C^{\prime}}{\partial y^{2}}$

The initial and boundary conditions are prescribed as:

$$
\begin{aligned}
& t^{\prime} \leq 0: u=0, v=0, T^{\prime}=T_{\infty}^{\prime}, C^{\prime}=C_{\infty}^{\prime} \text { for all } \mathrm{x}, \mathrm{y}, \\
& t^{\prime}>0: u=0, v=0, T^{\prime}=T_{w}^{\prime}, C^{\prime}=C_{w}^{\prime} \text { at } \mathrm{y}=0 \\
& u \rightarrow 0, T^{\prime} \rightarrow T_{\infty}^{\prime}, C^{\prime} \rightarrow C_{\infty}^{\prime} \text { at } \mathrm{x}=0, \\
& u \rightarrow 0, T^{\prime} \rightarrow T_{\infty}^{\prime}, C^{\prime} \rightarrow C_{\infty}^{\prime} \text { as } y \rightarrow \infty .
\end{aligned}
$$

where all the parameters defined in the nomenclature.

In the momentum (velocity boundary layer) Equation (7) the second term on the right hand side is the viscoelastic mixed derivative term, the third term is thermal buoyancy force, the fourth term is the species (concentration) buoyancy force, the penultimate term is the Darcian linear drag and the final term is the Forchheimer quadratic drag force. Now we implement the Rosseland approximation [1]. Refractive index of the fluid medium is constant. Unidirectional radiation flux, $q_{r}$, is considered and it is assumed that $\frac{\partial q_{r}}{\partial y} ? \frac{\partial q_{r}}{\partial x}$. This model is valid for optically-thick media in which thermal radiation propagates only a limited distance prior to experiencing scattering or absorption. The local thermal radiation intensity is due to radiation emanating from proximate locations in the vicinity of which emission and scattering are comparable to the location of interest. For zones where conditions are appreciably different, thermal radiation has been shown to be greatly attenuated before arriving at the location under consideration. The energy transfer depends on conditions only in the area adjacent to the plate regime i.e. the boundary layer regime. Rosseland's model is physically valid for intensive absorption i.e. optically-thick flows which are optically far from the bounding surface. Implicit in this approximation is also the existence of wavelength regions where the optical thickness may exceed a value of five. Under this approximation we arrive at the following expression for unidirectional radiative heat flux $q_{r}$ :

$q_{r}=-\frac{4 \sigma_{S}}{3 k_{e}} \frac{\partial T^{\prime}}{\partial y}$

where $\sigma_{s}$ is the Stefan-Boltzmann constant and $k_{e}$ is the mean absorption coefficient, respectively. The optically-thick assumption is realistic for certain polymeric fluids [9]. If temperature differences within the flow are sufficiently small, then Equation (11) can be linearized by expanding $T^{\prime 4}$ into the Taylor series about $T_{\infty}^{\prime}$, which after neglecting higher order terms takes the form:

${T^{\prime}}^{4} \cong 4{T_{\infty}^{\prime}}^{3} T^{\prime}-3{T_{\infty}^{\prime}}^{4}$

In view of Equations (11) and (12), Equation (8) reduces to

$$
\frac{\partial T^{\prime}}{\partial t^{\prime}}+u \frac{\partial T^{\prime}}{\partial x}+v \frac{\partial T^{\prime}}{\partial y}=\alpha \frac{\partial^{2} T^{\prime}}{\partial y^{2}}+\frac{16 \sigma_{s} T_{\infty}^{\prime}}{3 k_{e} \rho c_{p}} \frac{\partial^{2} T^{\prime}}{\partial y^{2}}+\frac{v}{c_{p}}\left(\frac{\partial u}{\partial y}\right)^{2}
$$


where all parameters are defined in the nomenclature.

Local skin-friction (wall shear stress), local Nusselt number and local Sherwood number are given respectively by:

$$
\begin{gathered}
\tau_{x}=-\mu\left(\frac{\partial u}{\partial y}\right)_{y=0} \\
N u_{x}=\frac{-x\left(\frac{\partial T^{\prime}}{\partial y}\right)_{y=0}}{T_{w}^{\prime}-T_{\infty}^{\prime}} \\
S h_{x}=\frac{-x\left(\frac{\partial C^{\prime}}{\partial y}\right)_{y=0}}{C_{w}^{\prime}-C_{\infty}^{\prime}}
\end{gathered}
$$

Proceeding with analysis, we implement the following nondimensional quantities to facilitate a numerical solution to the boundary value problem defined by Equations (6) to (9) under conditions (10) :

$X=\frac{x}{L}, Y=\frac{y}{L}\left(G r_{L}\right)^{\frac{1}{4}}, \quad R=\frac{r}{L}$, where $r=x \sin \phi$,

$V=\frac{v L}{v}\left(G r_{L}\right)^{\frac{-1}{4}}, U=\frac{u L}{v}\left(G r_{L}\right)^{\frac{-1}{2}}, t=\frac{v t^{\prime}}{L^{2}}\left(G r_{L}\right)^{\frac{1}{2}}$

Equations (6), (7), (13) and (9) are reduced to the following non-dimensional form

$\frac{\partial(U R)}{\partial X}+\frac{\partial(V R)}{\partial Y}=0$

$\frac{\partial U}{\partial t}+U \frac{\partial U}{\partial X}+V \frac{\partial U}{\partial Y}=\frac{\partial^{2} U}{\partial Y^{2}}-\Gamma \frac{\partial^{3} U}{\partial Y^{2} \partial t}+T \cos \phi$

$+N C \cos \phi-\frac{U}{D a G r_{L}}-\frac{F s}{D a} U^{2}$

$\frac{\partial T}{\partial t}+U \frac{\partial T}{\partial X}+V \frac{\partial T}{\partial Y}=\frac{1}{\operatorname{Pr}}\left[1+\frac{4}{3 F}\right] \frac{\partial^{2} T}{\partial Y^{2}}$

$\frac{\partial C}{\partial t}+U \frac{\partial C}{\partial X}+V \frac{\partial C}{\partial Y}=\frac{1}{S c} \frac{\partial^{2} C}{\partial Y^{2}}$

The corresponding initial and boundary conditions are

$t \leq \mathrm{O}: U=\mathrm{O}, V=\mathrm{O}, T=\mathrm{O}, C=\mathrm{O}$ for all $\mathrm{X}, \mathrm{Y}$,

$t>0: U=0, V=0, T=1, C=1$ at $\mathrm{Y}=0$,

$U=0, \quad T=0, \quad C=0$

at $\mathrm{X}=0$

$U \rightarrow 0, \quad T \rightarrow 0, C \rightarrow 0$

as $Y \rightarrow \infty$

The corresponding non-dimensional initial and boundary conditions are given by

$t \leq \mathrm{O}: U=\mathrm{O}, V=\mathrm{O}, T=\mathrm{O}, C=\mathrm{O}$ for all $\mathrm{X}, \mathrm{Y}$,

$$
\begin{gathered}
t>0: U=0, V=0, \frac{\partial T}{\partial Y}=-X^{m}, \frac{\partial C}{\partial Y}=-X^{n} \text { at } \mathrm{Y}=0, \\
U=0, \quad T=0, \quad C=0 \text { at } \mathrm{X}=0, \\
U \rightarrow 0, \quad T \rightarrow 0, \quad C \rightarrow 0 \quad \text { as } Y \rightarrow \infty .
\end{gathered}
$$

Where again all the parameters are given in the nomenclature The dimensionless local values of the skin friction, Nusselt number and the Sherwood number are given by the following expressions

$$
\begin{aligned}
& \tau_{X}=-\left(\frac{\partial U}{\partial Y}\right)_{Y=0} \\
& N u_{X}=-X\left(\frac{\partial T}{\partial Y}\right)_{Y=0} \\
& S h_{X}=-X\left(\frac{\partial C}{\partial Y}\right)_{Y=0}
\end{aligned}
$$

\section{NUMERICAL SOLUTION}

In order to solve the unsteady, non-linear, coupled equations (18)-(21) under the conditions (22), an implicit finite difference scheme of Crank-Nicolson type which is discussed in [10], [11], [12] and [13]. The finite difference scheme of dimensionless governing equations is reduced to tri-diagonal system of equations and is solved by Thomas algorithm as discussed in [14]. The region of integration is considered as a rectangle with $X_{\max }=1$ and $Y_{\max }=22$ where $Y_{\max }$ corresponds to $Y=\infty$ which lies very well out side both the momentum and thermal boundary layers. The maximum of $\mathrm{Y}$ was chosen as 22 , after some preliminary investigation so that the last two boundary conditions of (16) are satisfied within the tolerance limit $10^{-5}$. The mesh sizes have been fixed as $\Delta X=0.05, \Delta Y=0.05$ with time step $\Delta t=0.01$. The computations are carried out first by reducing the spatial mesh sizes by $50 \%$ in one direction, and later in both directions by $50 \%$. The results are compared. It is observed in all cases, that the results differ only in the fifth decimal place. Hence, the choice of the mesh sizes seems to be appropriate. The scheme is unconditionally stable. The local truncation error is $O\left(\Delta t^{2}+\Delta Y^{2}+\Delta X\right)$ and it tends to zero as $\Delta t, \Delta X$ and $\Delta Y$ tend to zero. Hence, the scheme is compatible. Stability and compatibility ensure the convergence.

\section{RESULTS AND DISCUSSION}

Only selective figures have been reproduced here for brevity. Default values of the thermophysical parameters are specified as follows: radiation parameter $(F)=3.0$ (strong thermal radiation compared with thermal conduction), viscoelasticity parameter $(\Gamma)=0.005$, buoyancy ratio parameter $(N)=1.0$, Schmidt number $(S c)=0.6$ (oxygen diffusing in the viscoelastic fluid), Prandtl number $(\operatorname{Pr})=0.7$ (water-based solvents), semi-vertical angle of the cone $(\phi)=$ $20^{0}$, Grashof number $\left(G r_{L}\right)=1.0$, Darcy number $(\mathrm{Da})=0.1$ (high permeability), Forchheimer number $(\mathrm{Fs})=0.1$ (weak quadratic drag). All graphs therefore correspond to these 
values unless otherwise indicated. In order to prove the accuracy of the computations in steady state

at $\mathrm{X}=1.0, \operatorname{Pr}=0.7, \eta=Y$ and considering

$G r_{L}^{*}=G r_{L} \cos \phi=\frac{g \beta \cos \phi\left(T_{w}^{\prime}-T_{\infty}^{\prime}\right) L^{3}}{v^{2}}$ are compared with available similarity solutions in the literature. The local skinfriction $\tau_{X}$ and local Nusselt number $N u_{X}$ values for different Prandtl number are compared with the results of isothermal case $(\mathrm{n}=0)$ of [15] and [16] in Tables 1, 2 respectively, and are found to be in good agreement

Table 1 Comparison of steady state local skin-friction values at $X=1.0$ with those of [15] and [16]

\begin{tabular}{|c|c|c|c|c|}
\hline \multirow{2}{*}{$\operatorname{Pr}$} & \multicolumn{2}{|c|}{ Hering[15] } & Pullepu Bapuji & \\
\cline { 2 - 3 } & \multirow{2}{*}{$f^{\prime \prime}(0)$} & $f^{\prime \prime}(0) \sqrt{\operatorname{Pr}}$ & et al.[16] & Present \\
& & & $\tau_{X} / G r_{L}^{3 / 4}$ & Results \\
\hline 0.03 & 7.185 & 1.2444 & 1.2406 & 1.24270 \\
\hline 0.1 & 3.466 & 1.0960 & 1.0932 & 1.09525 \\
\hline
\end{tabular}

\begin{tabular}{|l|l|l|l|l|}
\hline 0.7 & 0.9796 & 0.8195 & 0.8163 & 0.81312 \\
\hline 1.0 & 0.7694 & 0.7694 & 0.7660 & 0.76665 \\
\hline
\end{tabular}

Table 2 Comparison of steady state local Nusselt number values at $X=1.0$ with those of [15] and [16]

\begin{tabular}{|c|c|c|c|c|}
\hline \multirow[b]{2}{*}{$\operatorname{Pr}$} & \multicolumn{2}{|c|}{ Hering[15] } & \multirow{2}{*}{$\begin{array}{c}\text { Pullepu } \\
\text { Bapuji } \\
\text { et al.[16] } \\
N u_{X} / G r^{1 / 4}\end{array}$} & \multirow[b]{2}{*}{$\begin{array}{l}\text { Present } \\
\text { Results }\end{array}$} \\
\hline & $-\theta^{\prime}(0)$ & $-\theta^{\prime}(0) \sqrt{\operatorname{Pr}}$ & & \\
\hline 0.03 & 0.7185 & 0.12440 & 0.1258 & 0.12524 \\
\hline 0.1 & 0.6683 & 0.2113 & 0.2121 & 0.21202 \\
\hline 0.7 & 0.5392 & 0.4511 & 0.4554 & 0.45254 \\
\hline 1.0 & 0.5104 & 0.5104 & 0.5157 & 0.51461 \\
\hline
\end{tabular}
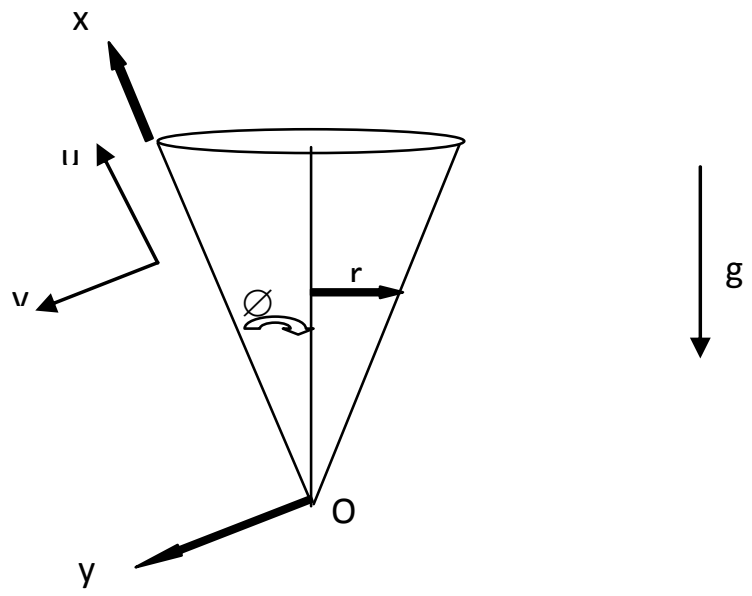

Fig 1: Physical Model

Figures 1(a) and 1(b) show the influence of radiation parameter, $F$, on steady state velocity $(U)$ and temperature $(T)$ distributions with distance into the boundary layer, transverse to the cone surface $(Y) . \quad F=\frac{k_{e} k}{4 \sigma_{s} T_{\infty}^{\prime 3}}$ and this defines the ratio of thermal conduction contribution relative to thermal radiation. For radiative heat transfer dominance in the boundary layer regime, $F \rightarrow 0$. For finite values of $\mathrm{F}$ there will be a simultaneous presence of thermal conduction and radiative transfer contributions. For $\mathrm{F}=1$, both modes will contribute equally. An increase in $F$ from 0 (total thermal radiation dominance) through $0.5,1.0,3.0,5.0,10.0$ to 100.0, causes a significant reduction in velocity with distance into the boundary layer i.e. retards the flow. With an increase in $F$ (decrease in thermal radiation contribution) the time taken to attain the steady state is markedly lowered. Increasing thermal radiation flux i.e. reduction in $F$, therefore delays the time 
taken to attain the steady state in the boundary layer regime. Temperature values $(T)$ are also considerably reduced with an increase in $F$ as there is a progressive decrease in thermal radiation contribution accompanying this. Figure 2 depict the response of the steady state temperature $(T)$ distributions to different values of the viscoelastic material parameter, $\Gamma$. An increase in $\Gamma$ from 0 to $0.001,0.003$ and to the largest value of 0.005 , decrease the temperature distributions accompanies an increase in $\Gamma$. In Figure 3, the distributions of steadystate streamwise temperature function $(T)$ versus spanwise coordinate $(Y)$ for various buoyancy ratio parameters $(N)$ close to the leading edge (i.e. cone apex) at $X=1.0$, are shown. The parameter $N=\frac{\beta^{*}\left(C_{w}^{\prime}-C_{\infty}^{\prime}\right)}{\beta\left(T_{w}^{\prime}-T_{\infty}^{\prime}\right)}$ defines the ratio of the species (mass diffusion) buoyancy force to the thermal (heat diffusion) buoyancy force. An increase in $N$ from 0 through 1 , 2, 3, 4 to 5 clearly decreases temperature $(T)$ throughout the boundary layer transverse to the cone surface and that again increasing $N$ is responsible for a decrease in the time taken to attain the steady-state. Figure $\mathbf{4}$ depict the distribution of streamwise concentration $(C)$ versus spanwise coordinate $(Y)$ for various Schmidt numbers $(S c)$. An increase in $S c$ from 0.1 (low weight diffusing gas species) through 0.5 (oxygen diffusing) to 1.0 (denser hydrocarbon derivatives as the diffusing species), 3.0 and 5.0, clearly strongly suppresses concentration levels in the boundary layer regime. An increase in Schmidt number effectively depresses concentration values in the boundary layer regime since higher $S c$ values will physically manifest in a decrease of molecular diffusivity $(D)$ of the viscoelastic fluid i.e. a reduction in the rate of mass diffusion. Figure 5 illustrate the spanwise spatial response of the flow variables to Prandtl number (Pr). $\mathrm{Pr}$ defines the ratio of momentum diffusivity to thermal diffusivity. $P r<1$ physically corresponds to cases where heat diffuses faster than momentum. $\operatorname{Pr}=0.7$ is representative of water-based solvents and $P r \gg 1$ e.g. 10, 20 corresponds to lubricating oils, polymers etc. With increasing $\operatorname{Pr}$ from 0.3 through $0.7,1.0,5.0,10.0$ to 20.0, temperature, $T$ as shown in figure, is markedly reduced throughout the boundary layer. Time taken to arrive at the steady state profiles for temperature, initially decrease with a rise in Prandtl number from 0.3 up to 1 ; thereafter however the trend is reversed and time elapsing to the steady state is increased with increasing Prandtl number from 1 through 5 to 10 . Figure 6 shows the spanwise spatial distributions of the velocity $(U)$ for different values of semi-apex cone angle, $\phi$. With wider cone angles i.e. a rise from 20 through 30 to 50 degrees, the velocity profiles are considerably reduced close to the cone surface i.e. the flow is strongly decelerated near the wall. Figure 7 show the variation of dimensionless Nusselt number (surface heat transfer gradient), $N u_{X}$ versus streamwise coordinate $(X)$ for various conduction-radiation parameters $(F)$. Increasing $F$ values strongly enhance the local Nusselt number, $N u_{X}$ values. Therefore with greater thermal radiation (lower $F$ ) $N u_{X}$ values are smaller i.e. heat transfer rates at the cone surface are suppressed with increasing thermal radiation. Again increasing $F$ (progressively greater thermal conduction heat transfer contribution) causes a decrease in the time needed to attain the steady state. In Figure 8 the variation of dimensionless local skin friction (surface shear stress), $\tau_{X}$, versus streamwise coordinate $(X)$ for various viscoelasticity parameters $(\Gamma)$ are shown. Shear stress is strongly elevated with increasing viscoelasticity (i.e. stronger elastic effects) i.e. the flow is accelerated. In Figure 9 the distributions of $S h_{X}$, versus streamwise coordinate $(X)$ for various Schmidt numbers $(S c)$ are depicted. With increasing $S c$, local Sherwood number, $S h_{X}$, is accentuated markedly along the cone surface. Again as described earlier, the time taken to attain the steady state is maximized for the lowest $S c$ value i.e. $t=7.76$ for $S c=0.1$.

In Figure 10, the influence of Grashof number $\left(G r_{L}\right)$ on steady state velocity $(\mathrm{U})$ distributions with $\mathrm{Y}$-coordinate are shown. It is observed that increasing $G r_{L}$ values from 0.1 through 1.0, 10.0, 50.0 and 100.0 clearly increases velocity. Figure 11 shows the transient profiles of dimensionless temperature $(\mathrm{T})$ distributions with $\mathrm{Y}$-coordinate at $\mathrm{X}=1.0$ for various values of Darcy number (Da). It is observed that increasing Da values from 0.001 through $0.01,0.1$ and 1.0 reduces the temperature. For various values of Forchheimer inertial drag parameter $(\mathrm{Fs})$ the transient profiles of dimensionless velocity (U) are depicted in Figure 12. It is observed that increasing Fs values from 0.1 through 1.0, 5.0, 10.0, 20.0 and 50.0 leads to a fall in the velocity. The time taken to reach the steady state increases as Fs increases.

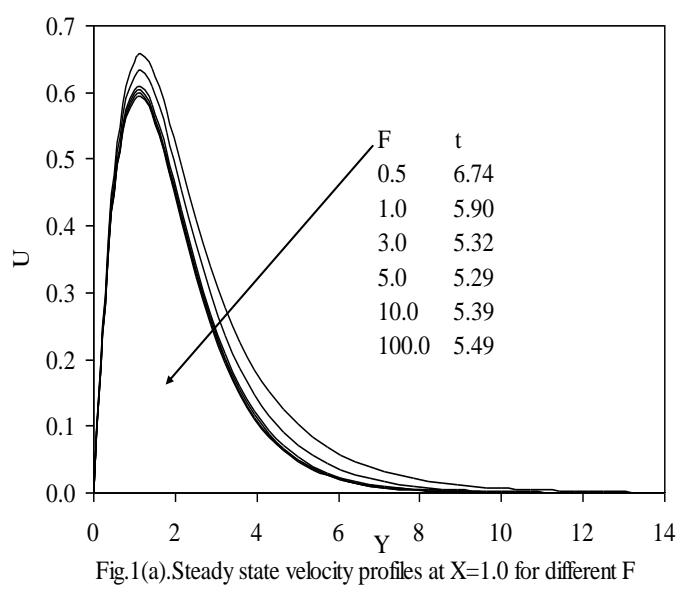




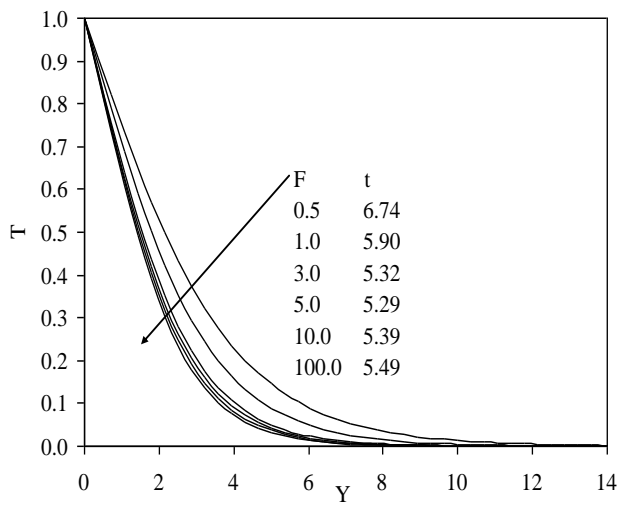

Fig.1(b).Steady state temperature profiles at $\mathrm{X}=1.0$ for different $\mathrm{F}$

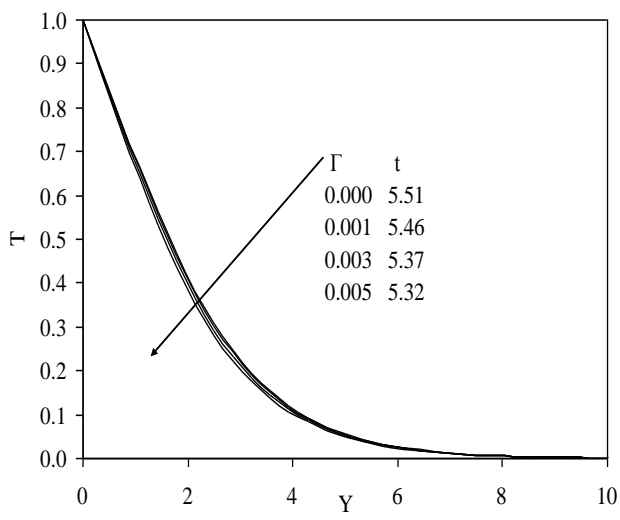

Fig.2.Steady state temperature profiles at $X=1.0$ for different $\Gamma$

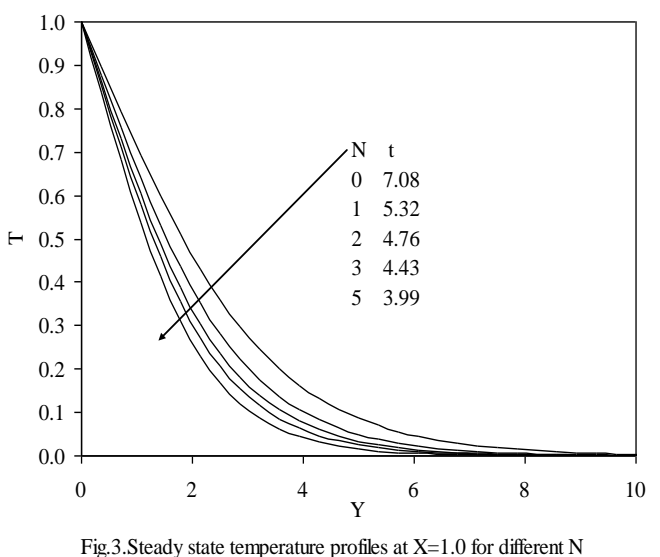

Fig.3.Steady state temperature profiles at $\mathrm{X}=1.0$ for different $\mathrm{N}$

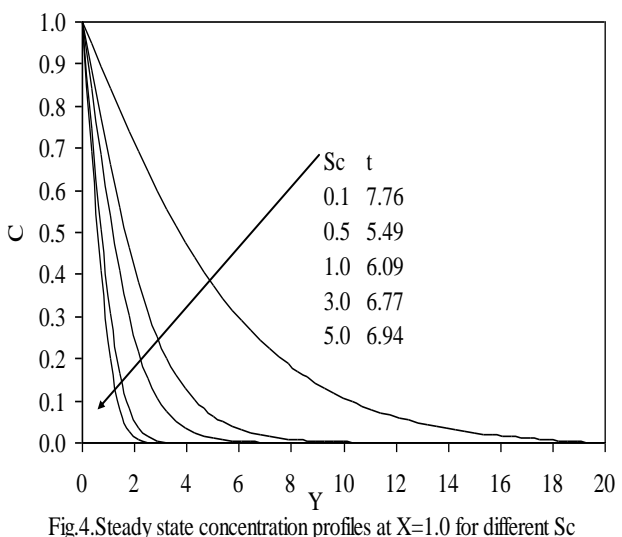

Fig.4.Steady state concentration profiles at $\mathrm{X}=1.0$ for different $\mathrm{Sc}$

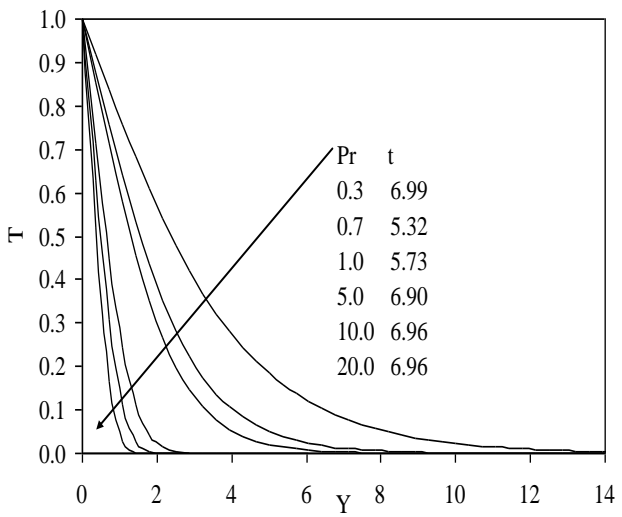

Fig.5. Steady state temperature profiles at $X=1.0$ for different $P r$
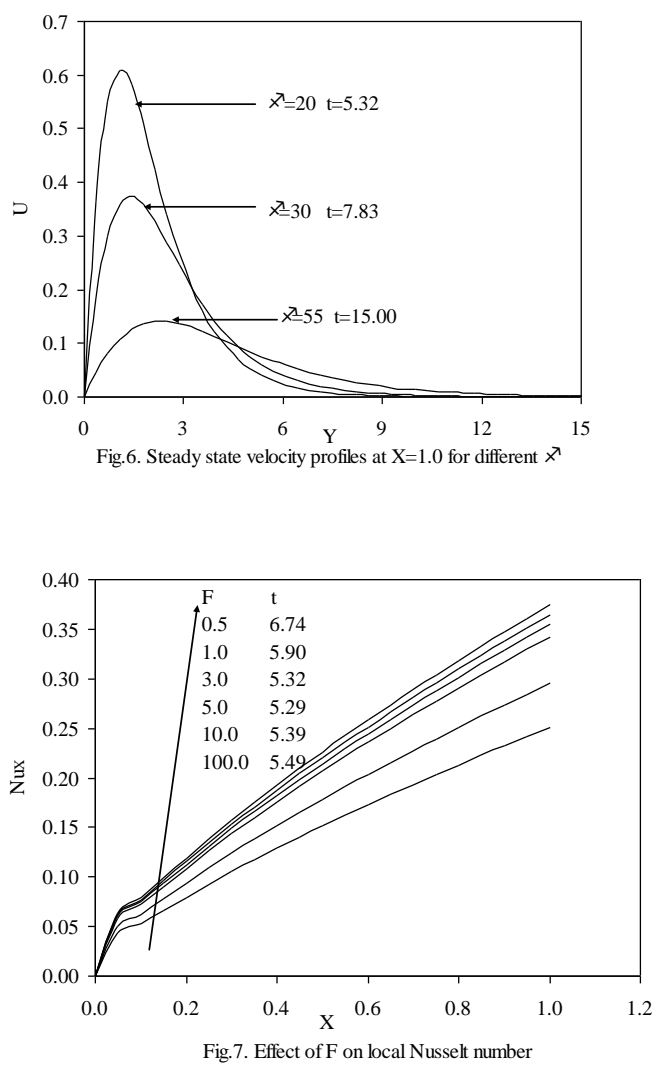

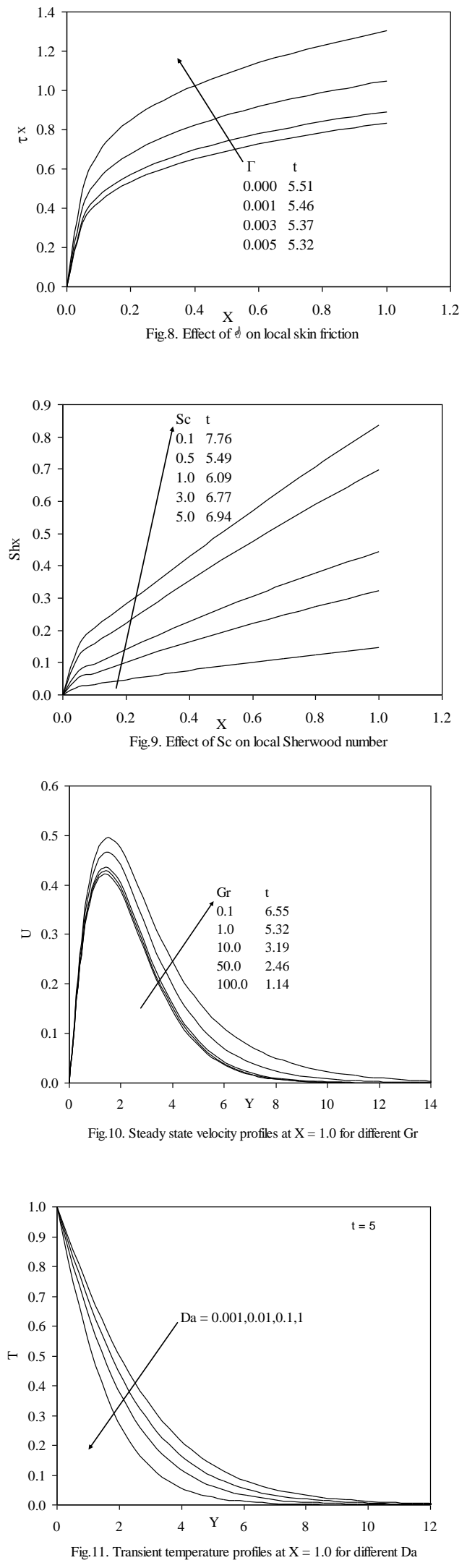

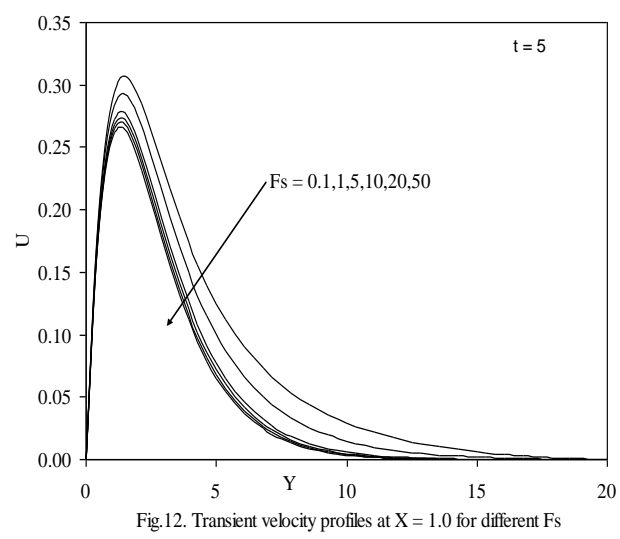

\section{CONCLUSIONS}

Numerical finite difference solutions have been developed for the transient laminar buoyancy-driven boundary layer heat and mass transfer in a Walters-B viscoelastic flow from a vertical stationary cone with appreciable thermal radiation effects.

- The computations have shown that increasing viscoelasticity $(\Gamma)$ accelerates the streamwise velocity and shear stress (local skin friction), local Nusselt number and local Sherwood number.

- Increasing thermal radiation (decreasing $F$ ) accelerates the flow and increases temperatures in the boundary layer regime but reduces local Nusselt number at the cone surface and also suppresses concentration values.

- An increase in cone semi-apex angle $(\phi)$ is observed to markedly decelerate the flow near the cone surface but to increase temperatures and concentrations in the boundary layer regime.

- Increasing buoyancy ratio parameter $(N)$ strongly accelerates the flow near the cone surface but suppresses temperature and concentration magnitudes.

- Increasing cone angle and thermal radiation also considerably increase the time taken to attain the steady state.

- Increasing the values of Darcian drag parameter (Da) or Grashof number $\left(G r_{L}\right)$ reduces the temperature and concentration but increase the velocity.

- It is observed that increasing the values of Forchheimer inertial drag parameter (Fs) leads to a fall in velocity but increases both the temperature and concentration.

- However increasing viscoelasticity and buoyancy ratio have a stabilizing effect on the transient regime and decrease the time required to attain the steady state.

The present model has applications in high temperature polymeric and other chemical processing technologies.

\section{REFERENCES}

[1] Raptis A. (1999). Radiation and viscoelastic flow, Int. Communications Heat Mass Transfer, 26, pp. 889-895. 
[2] Yih, K. A. (1999). Effect of radiation on natural convection about a truncated cone, Int $\mathrm{J}$ Heat Mass Transfer, 42, pp.4299-4305.

[3] Cheng, P., Le, T. T., Pop, I. (1985). Natural convection of a Darcian fluid about a cone, Int. J Heat Mass Transfer, 12, pp. 705-717.

[4] Yih, K. A. (1999). Coupled heat and mass transfer by free convection over a truncated cone in porous media: VWT/VWC or VHF/VMF, Acta Mechanica, 137, 83-97.

[5] Cheng, C. Y. (2000). An integral approach for heat and mass transfer by natural convection from truncated cones in porous media with variable wall temperature and concentration, Int. Commun. Heat Mass Transf, 27, pp. 537-548.

[6] Cheng, C. Y. (2009). Natural convection heat and mass transfer from a vertical truncated cone in a porous medium saturated with a non-Newtonian fluid with variable wall temperature and concentration, Int. Commun. Heat Mass Transf, 36, pp. 585-589.

[7] Barree, R.D., Conway, M. W. (2004). Beyond Beta Factors: A Complete Model for Darcy, Forchheimer, and Trans-Forchheimer Flow in Porous Media, paper SPE 89325 presented at the SPE ATCE held in Houston, TX, USA; 26-29 Sep.

[8] Walters, K. (1962). Non-Newtonian effects in some elastico-viscous liquids whose behaviour at small rates of shear is characterized by a general linear equation of state, Quart. J. Mech. Applied. Math., 15, pp. 63-76.
[9] Reeve, H.M., Mescher, A. M., Emery, A. F. (2004). Investigation of steady-state drawing force and heat transfer in polymer optical fiber manufacturing, ASME J. Heat Transfer, 126, pp. 236-243.

[10] Bapuji Pullepu, Ekambavanan, K., Chamkha, A. J. (2008). Unsteady laminar free convection from a vertical cone with uniform surface heat flux, Nonlinear Analysis: Modelling and Control, 13, pp. 47-60.

[11] Ganesan, P., Loganathan, P. (2001). Unsteady natural convection flow past a moving vertical cylinder with heat and mass transfer, Heat Mass Transf., 37, pp.59-65.

[12] Gouse Mohiddin, S., Computational Fluid Dynamics, LAP Lambert Academic Publishing, Germany 2011.

[13] Gouse Mohiddin, S., Prasad, V. R., Varma, S. V. K., Anwar Bég, O., Numerical Study Of Unsteady Free Convective Heat And Mass Transfer In A Walters-B Viscoelastic Flow Along A Vertical Cone, Int. J. of Appl. Math. and Mech., 6 (2010) 88-114.

[14] Carnahan, B., Luther, H. A., Wilkes, J.O. (1969). Applied Numerical Methods, John Wiley and Sons, New York.

[15] Hering, R. G. (1965). Laminar free convection from a non-isothermal cone, Int. J. Heat Mass Transfer, 8, pp.1333-1337.

[16] Pullepu, B., Ekambavanan, K., Chamkha, A. J. (2007). Unsteady laminar natural convection from a nonisothermal vertical cone, Nonlinear Analysis: Modelling and Control, 12, pp. 525-540. 